Article

\title{
In-Vitro Experimental Modeling of Oscillatory Respiratory Flow in a CT-Scanned OSAHS Tract
}

\author{
Zhenshan Zhu ${ }^{1}$, Yaping Ju ${ }^{1}$ and Chuhua Zhang ${ }^{1,2, *}$ \\ 1 Department of Fluid Machinery and Engineering, School of Energy and Power Engineering, \\ Xi'an Jiaotong University, Xi'an 710049, China; zzs2010@stu.xjtu.edu.cn (Z.Z.); \\ yapingju@mail.xjtu.edu.cn (Y.J.) \\ 2 State Key Laboratory for Strength and Vibration of Mechanical Structures, Xi'an Jiaotong University, \\ Xi'an 710049, China \\ * Correspondence: chzhang@mail.xjtu.edu.cn; Tel.: +86-029-866-8723
}

Received: 19 October 2020; Accepted: 9 November 2020; Published: 10 November 2020

\begin{abstract}
Obstructive sleep apnea-hypopnea syndrome (OSAHS) is a highly prevalent respiratory disorder. The knowledge of respiratory flow is an essential prerequisite for the establishment and development of OSAHS physiology, pathology, and clinical medicine. We made the first in-vitro experimental attempt to measure the oscillatory flow velocity in a computed tomography (CT) scanned extra-thoracic airway (ETA) model with OSAHS by using the particle image velocimetry (PIV) technique. In order to mimic respiration flow, three techniques were adopted to address difficulties in in-vitro experimental modeling: (1) fabricating the obstructive ETA measurement section with the CT-scanned data of an OSAHS patient airway; (2) maintaining the measurement accuracy by using the optical index-matching technique; (3) reproducing the oscillatory respiratory flow rates with the compiled clinical data of transient tidal volumes. The in-vitro measurements of oscillatory respiratory flow velocity manifested the time evolution of the complex OSAHS flow patterns, and the potential wall collapse of the ETA model with OSAHS.
\end{abstract}

Keywords: in-vitro experimental modeling; CT-scanned OSAHS model; oscillatory respiratory flow rates; particle image velocimetry technique; optical index matching

\section{Introduction}

The human airway is a key organ for the body to exchange air with the external environment. However, this passageway is susceptible to inhaled particulate matters, extreme atmospheric conditions, bad individual habits, and natural aging, resulting in a wide spectrum of respiratory diseases ranging from the simple common cold, snoring, nasal airway blockage and obstructive sleep apnea-hypopnea syndrome (OSAHS) [1]. In the case of such disorders, OSAHS is highly prevalent and poses a high risk of damaging human health and life [2-5]. Its severity is highly correlated to the flow characteristics of the upper airway [6]. Thus, the knowledge of OSAHS respiratory flow in the upper airway is of scientific significance for the establishment and development of OSAHS physiology and pathology, and of clinical medicine references for the diagnosis and therapy of OSAHS diseases.

Compared with the in-vivo measurement of respiratory flow in a realistic human airway, the in-vitro measurement of respiratory flow can effectively provide more detailed flow fields, allowing the respiratory physiologist to deepen the understanding of OSAHS pathology and the respiratory physician to improve diagnosis and therapy for OSAHS diseases. Nonetheless, OSAHS physiological studies of airway anatomy has earned much less attention than healthy airways. Only a small number of studies have focused on respiratory flow in OSAHS-like tract, although much experimental research on the respiratory flow has been carried out in simplified smooth multiple-generation airway 
models, which were briefly reviewed in Zhu et al. [7]. Following a series of numerical works on steady inspiratory or expiratory flow in smooth symmetrical and asymmetrical multiple-generation bifurcating airway models [8-10], Liu and his collaborators extended their numerical methods to unsteady respiratory flow in a human upper airway (HUA) model before and after OSAHS surgery [11]. Their numerical results confirmed that after surgery, the HUA flow resistance decreases with even more streamlined flow patterns, demonstrating the potential application of OSAHS respiratory flow in OSAHS surgery. Later, they numerically simulated the steady and unsteady flows in a HUA model with OSAHS resulting from nasopharyngeal obstruction [12]. Their numerical results indicated that the respiratory flow with OSAHS features strong flow injection caused by narrowing of the pharynx at both inspiration and expiration stages. Recently, Song et al. [13] numerically simulated steady inspiration flows in a nasopharyngeal obstruction model with OSAHS. Their model is quite similar to that used in Liu et al. [12]. The numerical results found that inspiratory flow velocity reached a maximum at the obstructed area with a strong negative pressure gradient. Very recently, Cui et al. [14] used the large eddy simulation (LES) method to investigate oscillatory respiration flows in an idealized extra-thoracic (ETA) airway. The simulated results indicated that the unsteady airflow structures are highly impacted by inspiration and expiration phases, and the techniques to model the breathing process.

As for the experimental measurements of respiratory flow, Kim and Chung [15] used tomographic particle image velocimetry (PIV) techniques to measure the airflow in disordered and corrected nasal cavity models. Later, Kim and his collaborators evaluated the feasibility of 3D steady tomographic PIV measurements in a normal nasal cavity [16]. More recently, Wu et al. [17] implemented a smoke-wire visualization of steady flow at the mid-sagittal plane of a HUA model with OSAHS by using a high-speed camera. Their experimental visualization, together with numerical simulation, suggested that for OSAHS patients, HUA narrowing is a symptom of OSAHS development and deterioration.

All of the above in-vitro experimental models for OSAHS respiratory flow were limited to steady flow cases. The recent numerical observations $[18,19]$ of the unsteady flow patterns differ significantly from the steady ones in the ETA models. In-vitro measurement of unsteady respiratory flow in human airway models poses a great challenge for fluid experimentalists due to related geometrical complexity, limited optical accessibility and oscillatory flow rate [20]. Specifically, three difficulties need to be overcome if an accurate and reliable mimicking of respiratory flow in in-vitro environment was required, i.e., a realistic respiratory tract model with OSAHS, good optical index matching, and reliable control over the oscillatory respiratory flow rate. It is worth noting that most investigations on oscillatory respiratory flow were based on the simplified sin curve flow rate as the oscillatory boundary condition $[18,19]$. In fact, the realistic oscillatory respiratory boundary condition is quite different from the sin function [21].

The goal of the present research is twofold. The primary goal is to tackle the above mentioned three critical difficulties arising in the in-vitro experimental modeling of OSAHS flows. The secondary goal is to employ the experimental rig to measure the oscillatory respiratory flows in an ETA model with OSAHS. The ETA model with OSAHS is composed of an oral cavity, pharynx, larynx and trachea with total closure of the nasal passageway.

The remainder of this paper is organized as follows. In Section 2, an in-vitro experimental model will be described, focusing on techniques to overcome the above three difficulties. Section 3 will present PIV techniques tailored to the experimental rig. Section 4 will present the PIV measurement results of oscillatory respiratory flow in the ETA model with OSAHS and their clinical implications. Concluding remarks will be made in Section 5.

\section{In-Vitro Experimental OSAHS Flow Model}

\subsection{ETA Model with OSAHS}

Creating an anatomically correct transparent experimental respiratory flow model is essential to measure respiration flow inside a human airway by using the PIV technique. The present ETA model 
was based an anatomical data, which was obtained from a scanned airway of a 48-year-old Chinese male OSAHS patient with a 128-slice spiral CT, under the rest breathing condition. The apnea-hypopnea index (AHI) and body mass index (BMI) were 52 and 26.8, respectively. In order to obtain detailed data, CT scanning was performed on the patient along the coronal, sagittal, and cross-sectional directions. The thickness between the CT-scanned slice images was $1 \mathrm{~mm}$. The OSAHS condition of the patient was so severe that the passageway through the nasal cavity was totally blocked in the retro uvula region (RUL) as shown in Figure 1. The ETA model composed of the oral cavity, pharynx, larynx and trachea was adopted to mimic the patient's breathing through the oral cavity. The ETA model with OSAHS was then constructed with the image processing software Mimics $[15,16,22]$. The digital ETA model is illustrated in Figure 2, from which the obstruction resulting from the retro uvula hypertrophy can be clearly observed in Figure 2a. Two representative measurement sections were selected, i.e., the global A-A section viewed from the lateral side and the local B-B section near the obstructed region, as shown in Figure 2a,b.

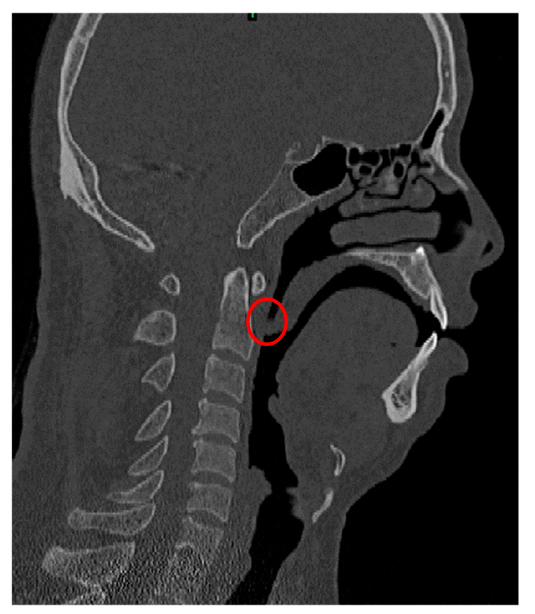

Figure 1. Sagittal plane CT image of OSAHS patient.

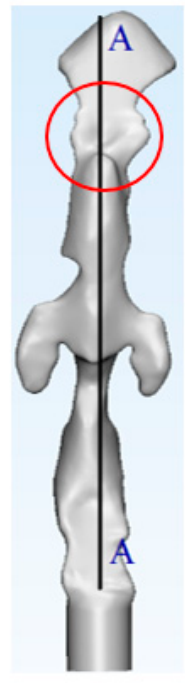

(a)

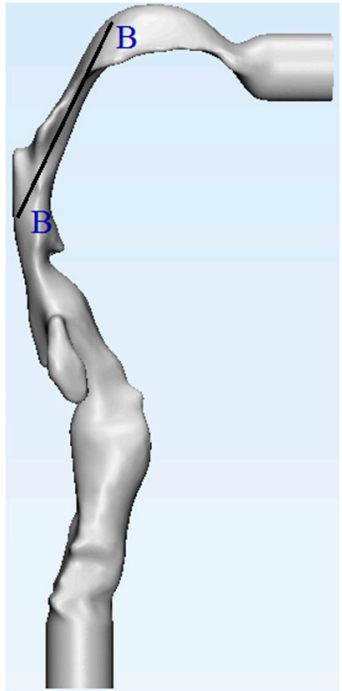

(b)

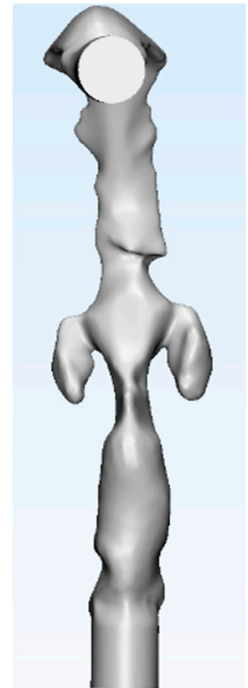

(c)

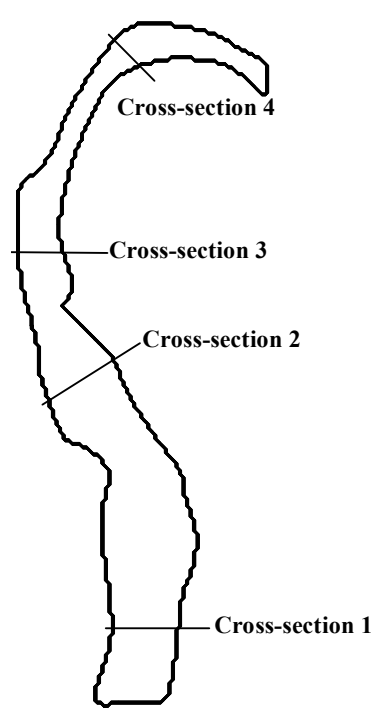

(d)

Figure 2. Reconstructed ETA model with OSAHS: (a) dorsal view; (b) lateral view; (c) front view; (d) four cross-sections.

To facilitate an accurate experimental measurement, the experimental model was finely fabricated as follows. First, a negative ETA model was printed with water-soluble material using a fused deposition 
modeling (FDM) printer $[16,23,24]$. The melting temperature of the water-soluble material was $220^{\circ} \mathrm{C}$ and the wire diameter was $1.75 \mathrm{~mm}$. The maximum printing error of the negative model was less than $0.1 \mathrm{~mm}$. Second, the negative model was coated with a water-soluble glue. The coating glue functioned as a slice separator of the negative model as the glue dried out. Both the water-soluble material and glue were mainly composed of polyvinyl alcohol (PVA), but they had different physical properties. The dried water-soluble glue will not stick to the external filler silicone, but the water-soluble material will. Third, the coated negative model was put into an optical glass box that was then carefully filled with transparent liquid silicone Sylgard 184 [25]. Before it was poured into the box, the silicone was needed to be vacuumed to remove bubbles in it. If not, the fabricated model wall would absorb tiny bubbles, which then seriously polluted measurement results. The liquid silicone was solidified by curing at room temperature over $48 \mathrm{~h}$. Finally, the negative model and the coating glue were washed with cold water. The fabricated experimental model is shown in Figure 3. The compliance of the human airway tract and the mucus attached to the inner wall $[26,27]$ were not considered in the present experimental model for the sake of accurate PIV measurements.

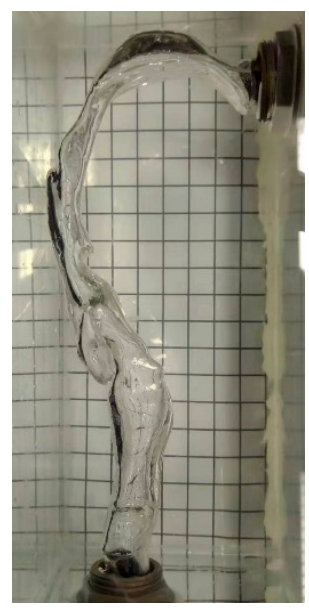

(a)

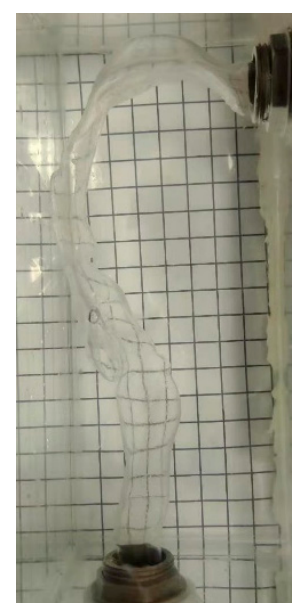

(b)

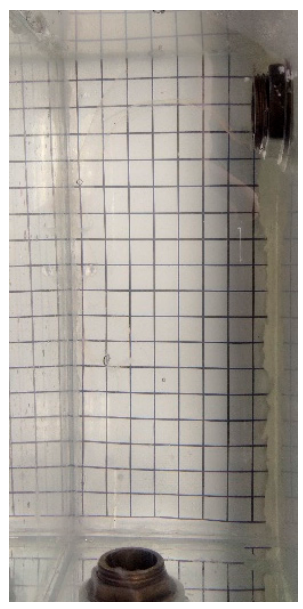

(c)

Figure 3. Optical index matching between ETA model and working fluids: (a) air; (b) water; (c) mixture of water and glycerol.

\subsection{Optical Index Matching}

In optical measurements of the flow inside the airway model, the optical index matching between the working fluid and model wall is crucial [20,28-31]. Good index matching can eliminate optical distortion to ensure measurement accuracy. After a series of priori tests with different mixtures of water and glycerol as working fluid, the mixture composed of $60.7 \%$ glycerol and $39.3 \%$ water [32] reached the best optical index matching. For comparison, Figure $3 a-c$ presents optical index matching with air, water and water-glycerol mixture, respectively, as working fluids in the model. Clearly, the best optical index match was achieved with the water-glycerol mixture. As shown in Figure 3c, the lines in the quadrille paper sheet do not suffer from deformation as one looks through the model [20].

\subsection{Oscillatory Respiratory Flow Rate}

In the present unsteady measurement, the realistic normal male breathing flow rate transients at rest were used to determine the modeling flow rate transients. Statistical data of the breathing flow rate transients compiled by a Beijing medical institution from hundreds of supine male adults were used. Similar flow rate transient curves were also confirmed in Kim and Chung [21] and Huang and Zhang [33]. The tide volume $\left(V_{\mathrm{T}}\right)$ here was chosen to be $500 \mathrm{~mL}$. The case where the inspiratory air volume is a little larger than the expiratory one [34] was not considered in the present test rig. 
The typical breathing cycle period at rest is within $3-5 \mathrm{~s} ; 5 \mathrm{~s}$ were chosen due to the severe status of the OSAHS patient.

To mimic the realistic flow physics and respiratory physiology, the similarities of Womersley number $\left(\alpha=L / 2 \cdot \sqrt{2 \pi \cdot f_{\mathrm{b}} / v}\right)$ and Reynolds number $(R e=V \cdot L / v)$ were kept between the breathing air and the modeling water-glycerol liquid $[23,29,32,35]$. The two similarity criteria ensured flow similarity such as the same relationship of pressure drop coefficient vs. flow coefficient. The Womersley number is a dimensionless number in biofluid mechanics representing the ratio of transient inertial force to viscous force and is an important similarity criterion for the oscillatory respiratory flows. Here, the Re number can be defined as $R e=4 f_{\mathrm{b}} \cdot V_{\mathrm{T}} /(L \cdot v)$, as mentioned by Janke et al. [23,24]. In these definitions, $L$, $V_{\mathrm{T}}, f_{\mathrm{b}}$ and $v$ refer to the inlet hydraulic diameter of the tract, tide volume, frequency of the oscillatory respiration, and kinematic viscosity of the working fluid, respectively. The kinematic viscosity $(v)$ of the water-glycerol mixture and breathing air was $8.933 \times 10^{-6} \mathrm{~m}^{2} / \mathrm{s}$ and $15.03 \times 10^{-6} \mathrm{~m}^{2} / \mathrm{s}$, respectively, at room temperature during the experiments. In this oscillatory respiratory flow, the maximum Reynolds numbers during inspiration and expiration phases were 1442 and 1149, respectively, and the Womersley number was 2.89. To maintain the similarity of Reynolds number and Womersley number, the breathing cycle period $\left(1 / f_{\mathrm{b}}\right)$ of $5 \mathrm{~s}$ was tuned to be $8.5 \mathrm{~s}$ in the present in-vitro experimental model where the working fluid was taken as the water-glycerol liquid. The breathing flow rate transients as well as those employed in the experiment are shown in Figure 4. Under such oscillatory boundary condition, roles of inlet and outlet of this model would switch periodically. At the inspiration stage, the boundary at the mouth side can be considered as the inlet, and the boundary at the trachea side was the outlet. While at the expiration stage, the contrary would be the truth.

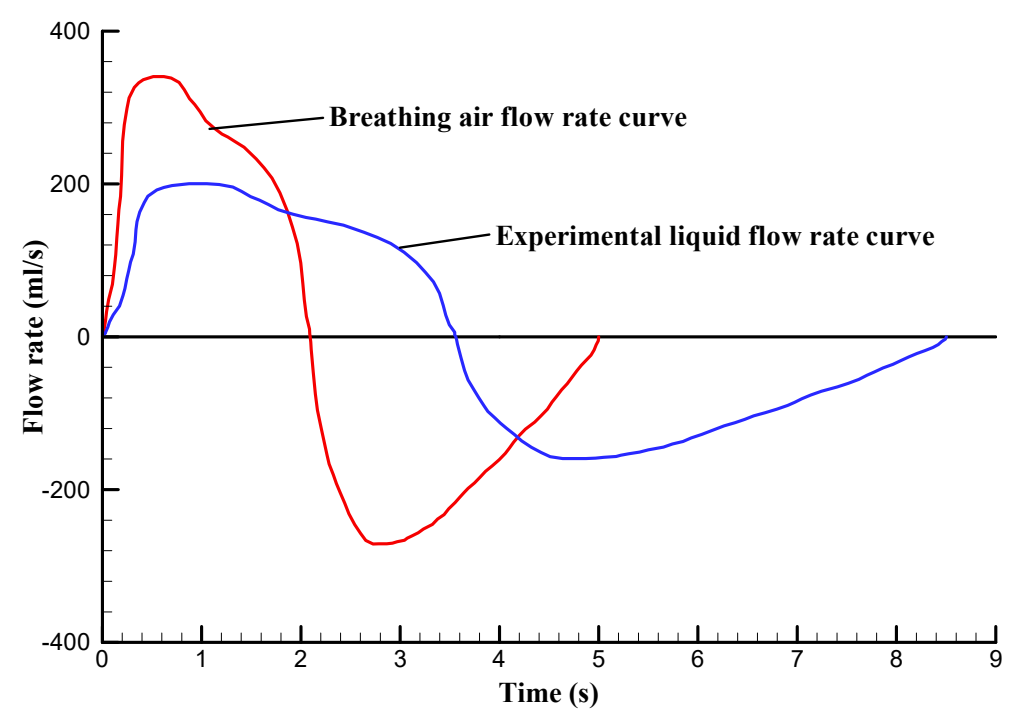

Figure 4. Human breathing and modelling flow rate transients.

To mimic the modeling flow rate transients as shown in Figure 4, several apparatuses and procedures were carefully designed in this research. The schematic and photo of the experimental setup are presented in Figure 5. A programmable logic controller (PLC), servo-motor, servo-motor driver, gear box, bent axle, piston, air exhausting device, reservoir and $V_{\mathrm{T}}$ monitor (Figure 5a) were employed to provide precise control over piston movements and thus experimental flow rate transients. Figure $5 b, c$ presents a photo of the main apparatus and experimental rig, respectively. First, a PLC program was developed and run on a personal computer to transfer the PLC input digital signal into the PLC output analog voltage signal. Second, step by step, the driver of the servo-motor drove the movements of the servo-motor. The duration of the movement in each step was $20 \mathrm{~ms}$. Thus, a total of 425 step movements were precisely controlled over the present single respiratory cycle (8.5 s). Third, the gear box was used to increase the servo-motor torque output, which eventually 
actuated the bent axle and the piston. Lastly, the air exhausting device was used to remove potential air bubbles absorbed into the test rig. The apparatus of the reservoir and $V_{\mathrm{T}}$ monitor was mainly made of a graduated cylinder, as shown in the Figure $5 \mathrm{~b}$. This cylinder can not only collect working fluids and add tracer particles, but also monitor the output $V_{\mathrm{T}}$. The liquid level in the graduated cylinder would fluctuate up and down, following simultaneously with the oscillatory experimental boundary. By recording the locations of the highest and lowest liquid levels in the graduated cylinder, the output $V_{\mathrm{T}}$ was monitored.

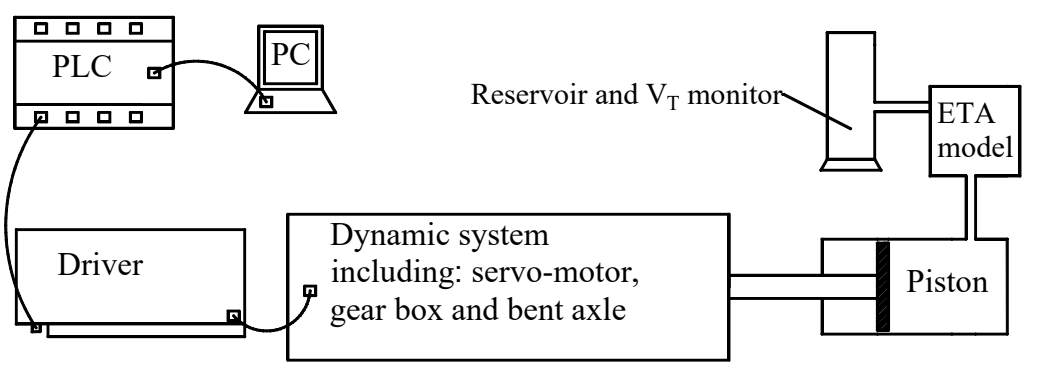

(a)

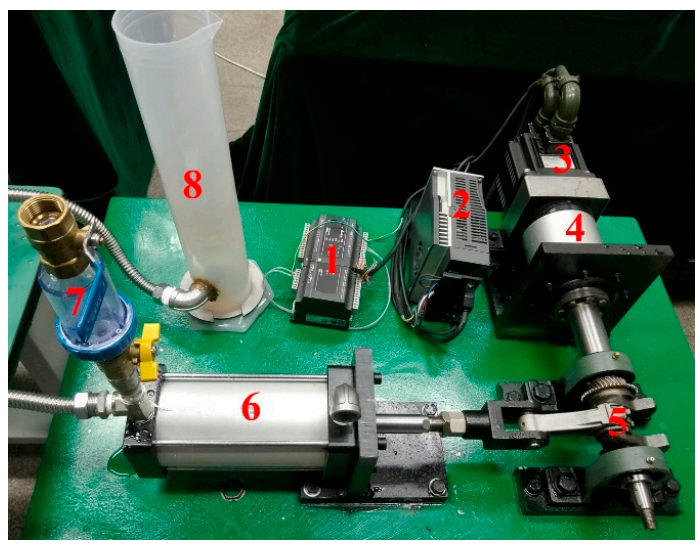

(b)

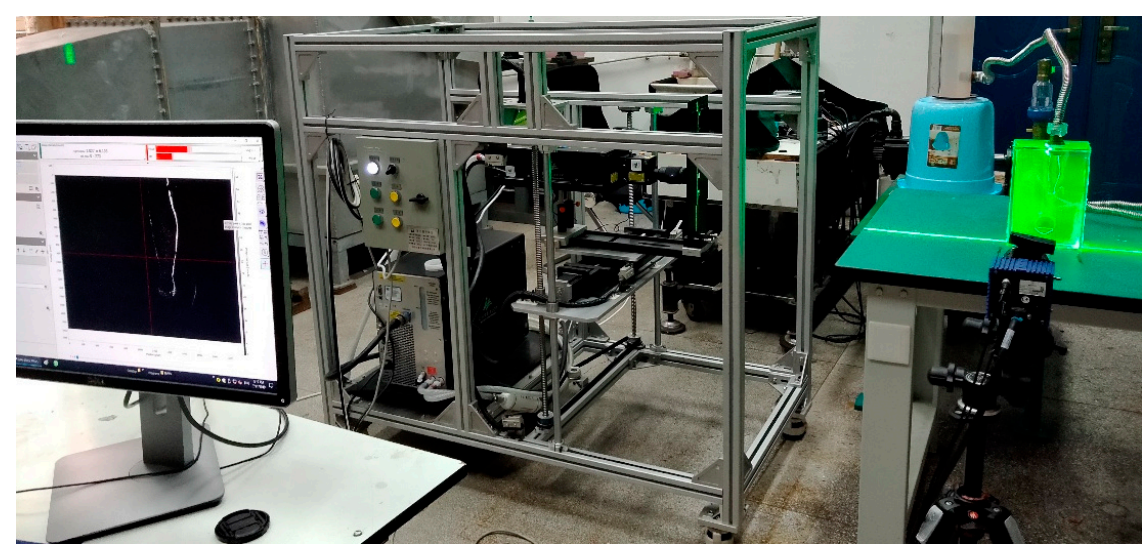

(c)

Figure 5. Experimental rig: (a) schematic; (b) main apparatus (except ETA tract and PIV system):1 PLC; 2 driver; 3 servo-motor; 4 gear box; 5 bent axle; 6 piston; 7 air exhausting device; 8 reservoir and $V_{\mathrm{T}}$ monitor; (c) photo of experimental rig. 


\section{PIV Techniques for the ETA Model with OSAHS}

The other goal of this research was to apply the above experimental rig to measure the oscillatory respiratory flow velocity in an ETA model with OSAHS. The LaVision 2D-2C (two-dimensional, two-component) PIV system was used to measure the unsteady flow velocity at the two representative sections, i.e., section A-A and section B-B, as shown in Figure 2a,b.

A double pulse yttrium-aluminum-garnet (YAG) laser and a high-speed charge-coupled device (CCD) camera were employed to obtain digital images. A laser (Evergreen Corp.) with power up to $600 \mathrm{~mJ}$ per pulse was used to provide double laser sheets with frequency of $15 \mathrm{~Hz}$, wavelength of $532 \mathrm{~nm}$ and thickness of approximately $1 \mathrm{~mm}$ to induce fluorescence of tracers. The CCD camera (Imager SX 4M) was used to store digital images. The camera features an interline transfer chip with progressive-scan low-noise readout, high spatial resolution of $2360 \times 1776$ pixels (4 Mpixel, $5.5 \times 5.5 \mu \mathrm{m}^{2}$ per pixel size) in 12-bit mode, and fast frame rates up to $31 \mathrm{~Hz}$. Here, $15 \mathrm{~Hz}$ frame rates were used to match the frequency of the laser pulse.

Determining the distinctive time $(d t)$ delay of two subsequent particle images (double shutter) in the PIV measurements of oscillatory flow is an important task since the $d t$ setting must be adapted to the maximum flow speed as well as the flow reversal condition [32]. Here, $d t$ was determined according to the CCD spatial resolution, field of view of the camera, estimated average velocity, and target pixel shift of tracing particles in double shutter, which were $2360 \times 1776$ pixels, $15 \times 11.3 \mathrm{~cm}^{2}, 0.7 \mathrm{~m} / \mathrm{s}$, and 7 pixels, respectively. The $d t$ and the duration of exposure time of particle images were determined to be $500 \mu \mathrm{s}$ and $200 \mu \mathrm{s}$, respectively. The length of camera image $(11.3 \mathrm{~cm})$ was smaller than that in ETA model (approximately $20 \mathrm{~cm}$ ); consequently, one PIV measurement of A-A section had to be divided into two sub-sections. The two digital images obtained from the two sub-sections measurements at the same phase were then stitched to form a whole PIV measurement image. Within the present modeling cycle ( $8.5 \mathrm{~s}), 128$ groups of double shutter images were continuously acquired with the PIV system and controlled with a synchronizer. All steps of control, acquisition and post processing were carried out in a DaVis 10.0.5 software environment [36].

The reflected light is very harmful to both the camera CCD itself and the CCD acquisition quality of near-wall flows. To avoid light reflection from walls, good optical index-matching, suitable tracer fluorescence and a long-pass glass filter were employed [16]. The working fluid with good optical index-matching not only eliminates optical distortions, but also reduces wall light reflection. Fluorescent tracers were used to scatter $590 \mathrm{~nm}$ wavelength light under $532 \mathrm{~nm}$ wavelength laser irradiation. Using the long-pass glass filter before the camera, the reflected light with wavelength below $550 \mathrm{~nm}$ will be filtered out, thus greatly alleviating wall reflection.

The long-pass glass filter and fluorescent tracers used in PIV measurement will not distort measured results. The long-pass glass filter had more than $99 \%$ transmittance of the scattered light with wavelength above $550 \mathrm{~nm}$. The mean diameter $d_{\mathrm{p}}$ and density $\rho_{\mathrm{p}}$ of fluorescent tracers were $4 \mu \mathrm{m}$ and $3.4 \mathrm{~g} / \mathrm{cm}^{3}$, respectively. The response time $\left(\tau_{\mathrm{p}}\right)$ of tracing particles defined as $d_{\mathrm{p}}{ }^{2} \rho_{\mathrm{p}} /(18 \mu)$ was calculated as $2.93 \times 10^{-7} \mathrm{~s}$ [30]. The dynamic viscosity $\mu$ of the water-glycerol mixture was $1.03 \times 10^{-2} \mathrm{~Pa} \cdot \mathrm{s}$.

\section{Results and Discussion}

In a respiration cycle, four representative phases were highlighted to present PIV measurements of oscillatory flow velocity fields at two typical A-A and B-B sections, as shown in Figure 6. Locations of A-A and B-B sections are defined in Figure 2a,b. The four selected phases represent the reversal phase of expiration-inspiration (phase one), the peak phase of inspiration (phase two), the reversal phase of inspiration-expiration (phase three), and the peak phase of expiration (phase four), respectively. The measured flow velocity contours were colored with the magnitude of absolute velocity (ABS velocity). The measured results will be presented and discussed in the following two sub-sections for reversal phases (phases one and three) and peak phases (phases two and four). For a clear presentation, local views at B-B sections were deliberately enlarged. In order to quantitatively analyze measured results, the main-stream 
velocity transients at four cross-sections were also presented. The locations and the main-stream velocities profiles of the four cross-sections are shown in Figures $2 \mathrm{~d}$ and 7 , respectively.

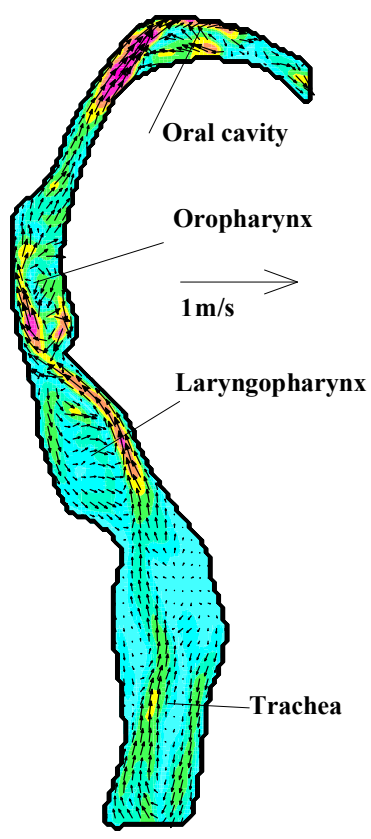

A-A section

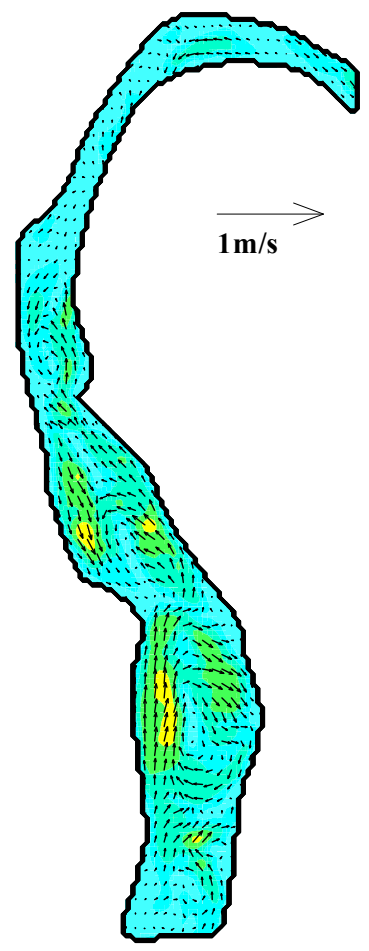

A-A section

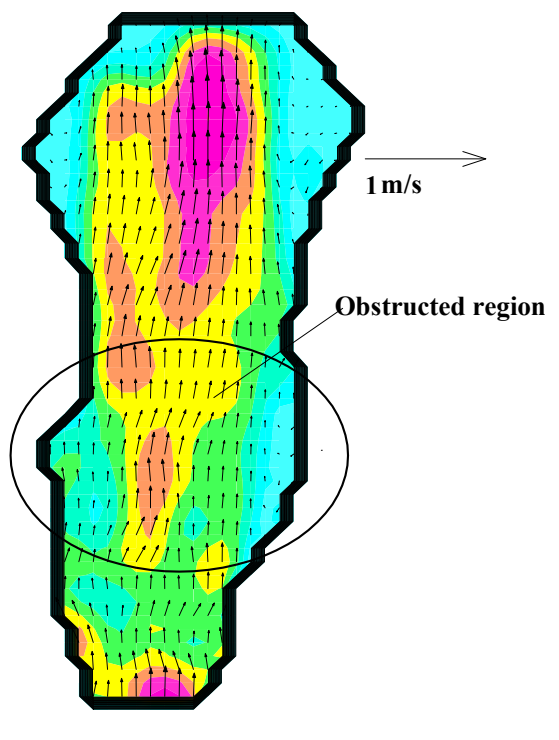

B-B section

(a)

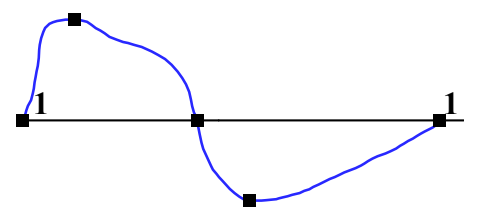

ABS Velocity [m/s]

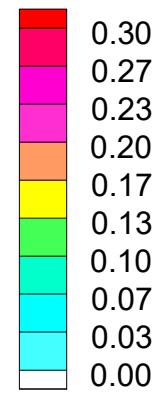

(c)
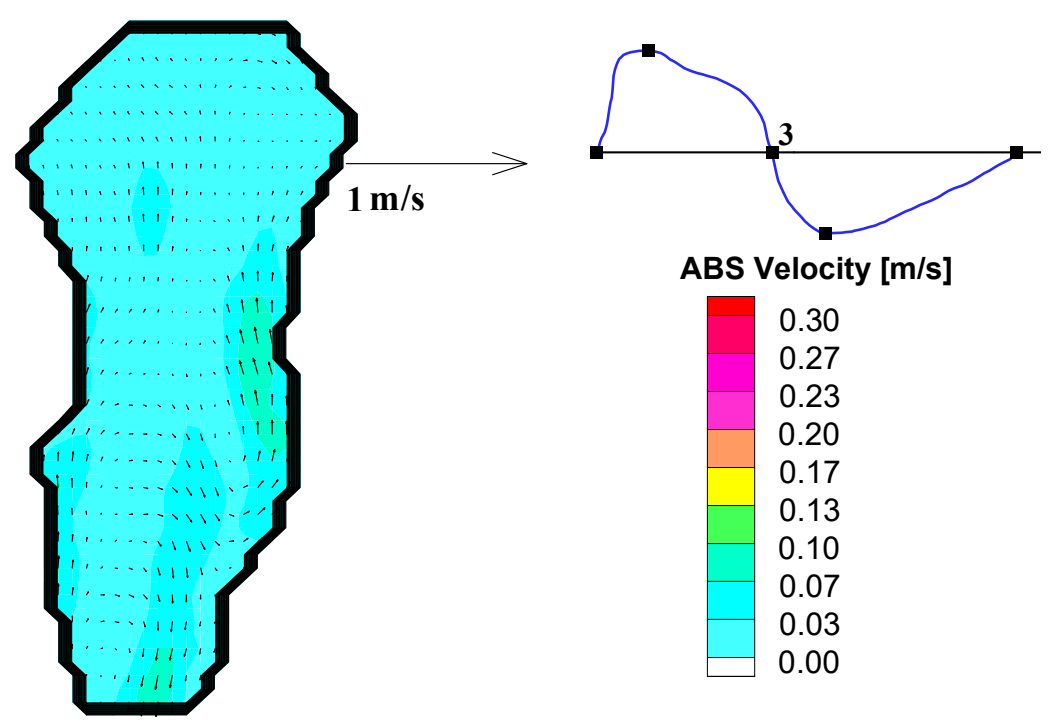

B-B section

(c)

(b)

Figure 6. Cont. 


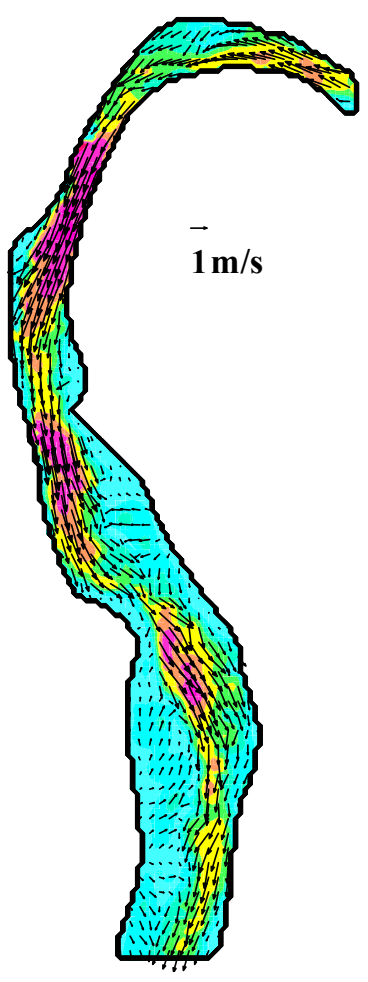

A-A section

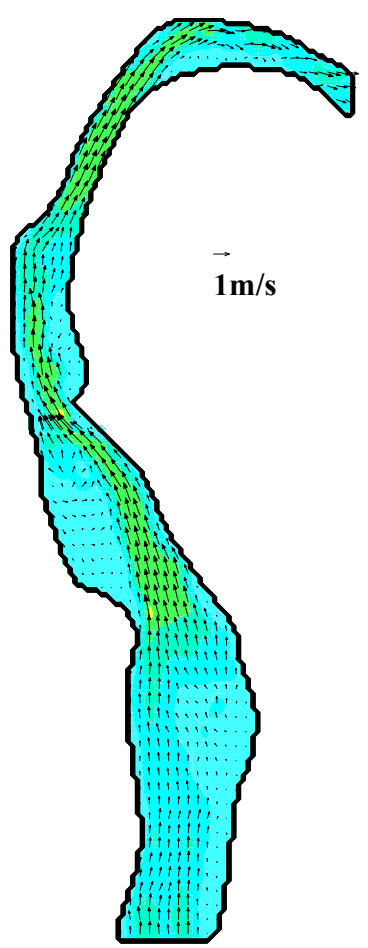

A-A section

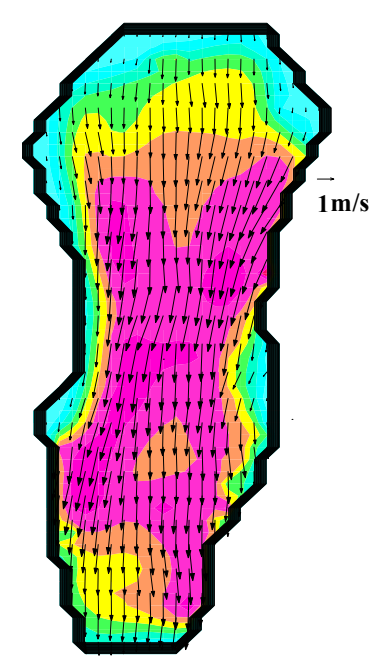

B-B section

(c)

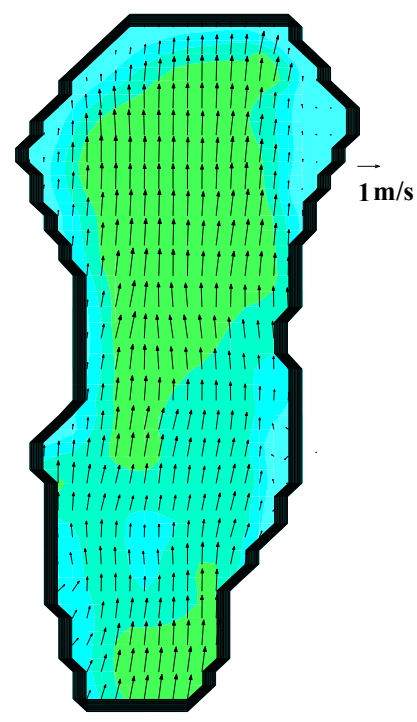

B-B section

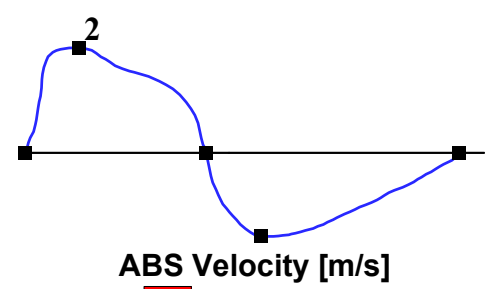

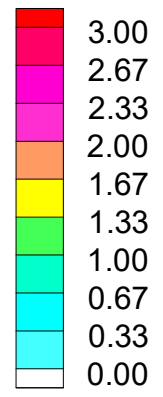

(c)
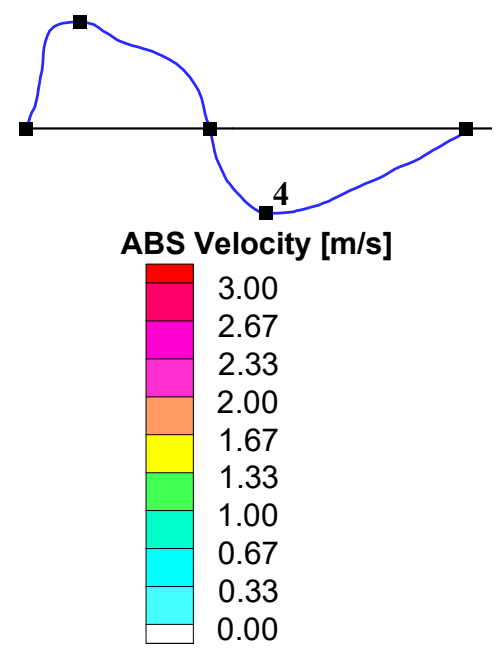

(d)

Figure 6. Measured results at four representative phases at A-A section and B-B section: (a) phase one: expiration-inspiration reversal transient; (b) phase three: inspiration-expiration reversal transient; (c) phase two: peak inspiration transient; (d) phase four: peak expiration transient. 


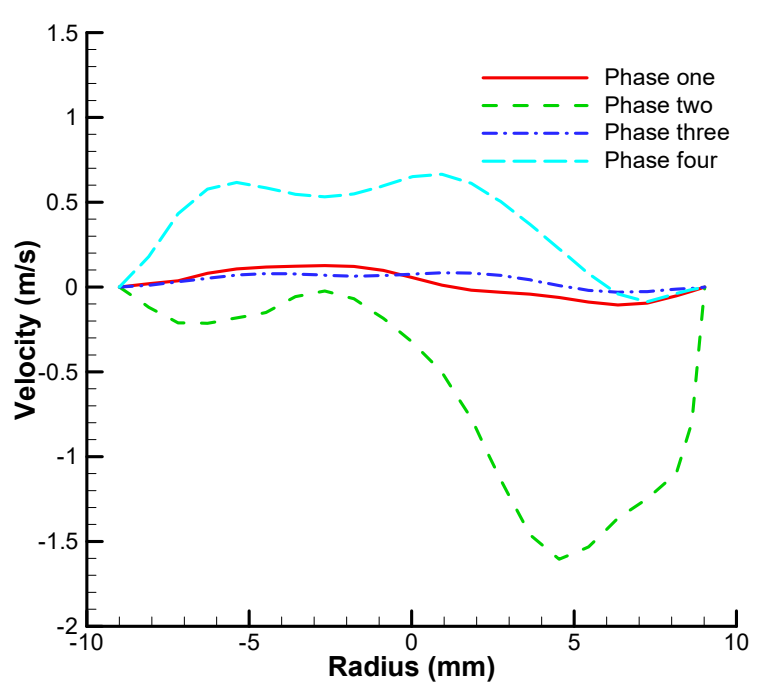

(a)

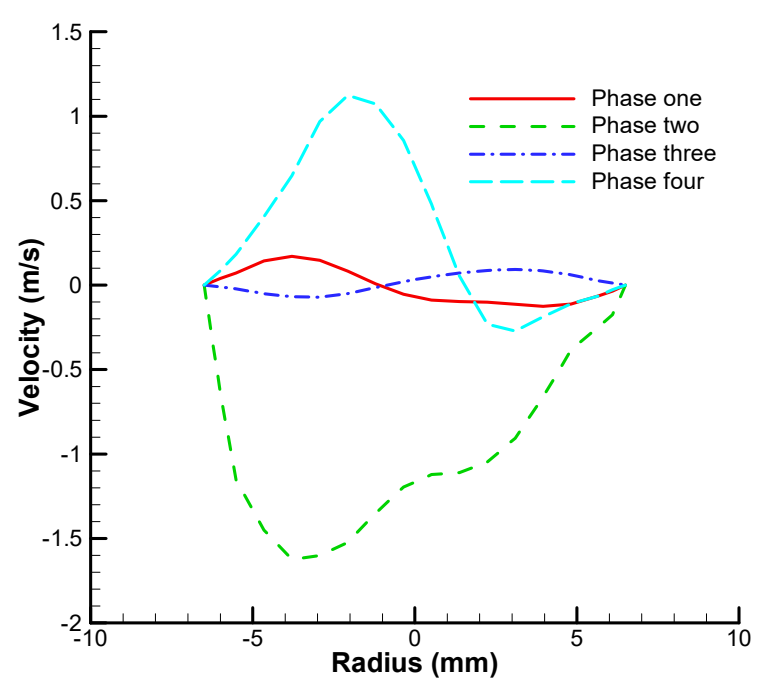

(c)

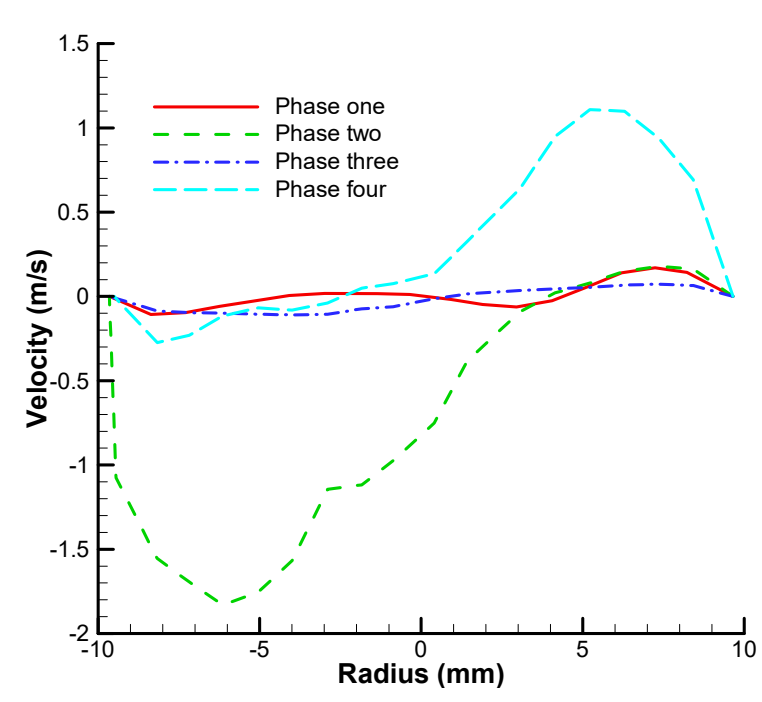

(b)

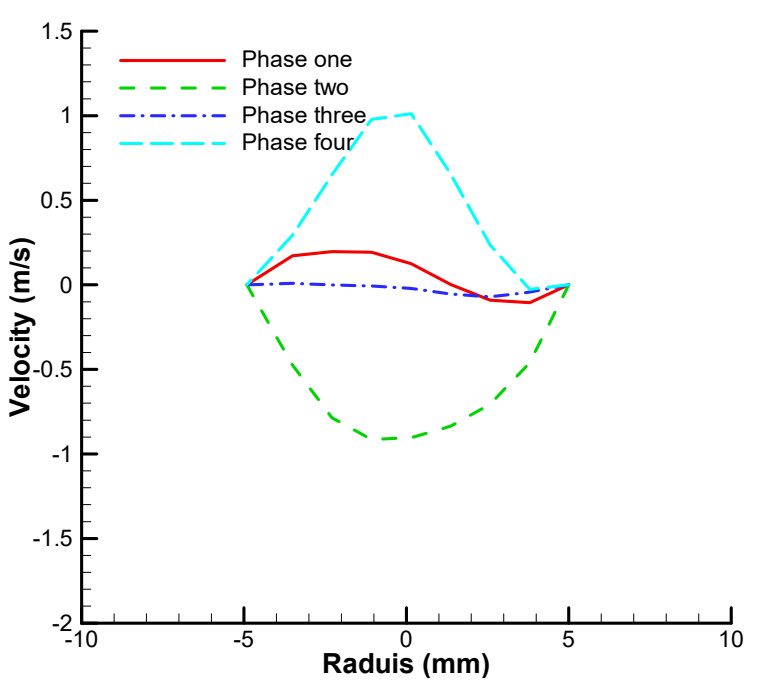

(d)

Figure 7. Measured main-stream velocities at four cross-sections at four representative phases: (a) cross-section 1; (b) cross-section 2; (c) cross-section 3; (d) cross-section 4. Locations of these four cross-sections are defined in Figure $2 \mathrm{~d}$.

\subsection{Reversal Phases}

At phase one, the respiratory flows are undergoing transition from expiration to inspiration (Figure 6a). The flow patterns presented are irregular both at the A-A section and the B-B section. The reversal flow and jet flow structures can be clearly observed at the A-A section. The interaction between the expiration and inspiration flows leads to strong flow reversal at the oral cavity and oropharynx region, which is hardly considered as either expiration or inspiration pattern. However, the local flow structure at the B-B section mainly corresponds to the expiration pattern with a large portion of expiration flow at the mainstream region, except only a small portion of reversal flow at the near wall region. The flow pattern at the expiration-inspiration phase is more complex than that observed in Adler and Brücker [22], who claimed that the flow at this phase could be regarded as expiration flow. This difference may be caused by more realistic oscillatory flow rate boundary conditions used in this research rather than the simplified sine-law flow rate boundary conditions used in Adler and Brücker [22]. Such jet-reversal flow structure is an indicator of complex 
expiration-inspiration transition at the quasi-static conditions where the net respiratory flow rate is zero while the unsteady inertial force and thus the respiratory flow acceleration and velocity are not zero.

We can also see that compared with the quasi-static condition at phase one, the jet-reversal flow structure occurs even more obviously at phase three (Figure $6 \mathrm{~b}$ ), due to the milder transition from inspiration to expiration (Figure 4). Neither an inspiration nor an expiration pattern is the dominating flow pattern at phase three. The jet-reversal flow structure extends into the oral cavity, oropharynx, laryngopharynx and trachea (Figure $6 \mathrm{~b}$, A-A section), and the widely spread reversal flow vortexes at the obstructed region (Figure $6 \mathrm{~b}, \mathrm{~B}-\mathrm{B}$ section). The inspiration-expiration pattern is very different from that in Adler and Brücker [22], who considered the flow at this phase to can be regarded as inspiration flow.

From the reversal transients, we could conclude that the realistic oscillatory respiratory boundary condition and the realistic model have a significant effect on respiration pattern, and associated flow structures. This observation can be clearly confirmed in Figure 7. Although the main-stream velocity profiles at reversal phases one and three are relatively small, their patterns are not similar to those at inspiration phase two and expiration phase four. Although quite few numerical and experimental studies focused on the reversal transients, the present PIV measurements confirm that the respiration patterns at the reversal phases can neither be regarded as the pattern of inspiration nor that of expiration due to the occurrence of jet-reversal flow and quasi-static condition.

\subsection{Peak Phases}

At peak inspiratory transient (phase two), the inspiration flows form an obvious jet-wake structure and a strong flow reversal (Figures $6 \mathrm{c}$ and $7 \mathrm{~b}$ ). Jet-wake flow structure, which often occurs in the human trachea and bronchi due to wall curvature [7], also appears at this phase in the ETA model with OSAHS. High-speed jet fluids accumulate at the dorsal side of the laryngopharynx region, while the anterior side of the trachea (Figure $6 c$, A-A section, Figure 7a,b). The narrowed tract does have a significant impact on inspiration flow at this phase with a clear injection phenomenon. As shown in the enlarged vector diagrams at the phase of peak inspiration, the jet-wake structure together with the flow reversal enhances fluid mixing and heat transfer, which benefits the velocity, temperature and humidity conditioning of the inhaled air as confirmed by Kim et al. [37].

At the peak expiration phase (phase four), however, the expiration flow shows very different flow patterns (Figure $6 \mathrm{~d}$ ). The high speed jet fluids accumulate at the anterior side of the laryngopharyngeal region and the dorsal side of the trachea (Figure $6 \mathrm{~d}$, A-A section, Figure $7 \mathrm{a}, \mathrm{b}$ ), which are at opposite sides of the tract at the peak inspiration phase (Figure 6c, A-A section). Such differences may be due to the combined effect of convection and viscous motions in the curved tract and complex wall attachments. The injection phenomenon caused by the narrowed region resembles the numerical observation in Liu et al. [12]. It should be noted that we used the oropharyngeal model in our research rather than the nasopharyngeal model used in Liu's study. The flow patterns at the trachea region are very different with the results in Wang et al. [38] using a simplified model and the results in Cui et al. [14] using an idealized model. In those studies, the expiration flow patterns at the trachea region were quite regular and presented straight tube-like flow. The differences may be caused by the more realistic model used in this research.

From the results above, we can clearly conclude that the inspiration and expiration flow patterns are quite different from each other. High speed jet fluids appeared at the opposite sides of the oropharynx, laryngopharynx and trachea regions, which was not highlighted in the previous similar numerical studies $[6,14,19,26,37]$. Expiration process is not a simple reversal process of inspiration.

Considering that the patient's nasal passageway was completely blocked, we believe that the collapse of the passageway most likely occurs at the peak inspiration phase at the obstructed region, thus resulting in apnea. There are two main reasons for this: firstly, the effect of obstructed tract on oscillatory respiratory flow fields at peak phases is much more apparent than that at the reversal phases. The occurrence of injection phenomenon at the narrowing obstructed area at the peak inspiration 
phase indicates very large pressure differences over this short, obstructed distance [13]. Secondly, at inspiration phase, the driving force induced by the movements of the thoracic cavity and abdominal cavity is a negative pressure. The combination of large pressure difference across the obstructed area and negative pressure at the peak inspiration phase would more easily induce collapse of passageway wall and eventual blockage of the tract, thus resulting in apnea. This condition worsens and becomes more dangerous for the deep-sleeping OSAHS patient, whose passageway tissue walls become very loose.

Overall, we presented a temporal and spatial evolution of the oscillatory respiratory flow patterns within a CT-scanned model with OSAHS using PIV measurements. It should be recognized that the calibrated CFD (computational fluid dynamics) method can provide even more flow details that are hardly, if not impossible, captured with PIV techniques [39], such as pressure drop and airway resistance in the CT-scanned complex geometry. Airway compliance is another important issue in human tract [40] that was not modeled in the present study. However, the present work contributes to an experimental modeling of the complex oscillatory respiratory flow by using PIV measurements and can be regarded as a step-stone for an even deeper understanding of OSAHS airway flows.

\section{Conclusions}

In the present research, particular efforts were made to experimentally mimic the respiratory flow conditions in an in-vitro experimental model to measure the oscillatory respiratory flow velocity inside a CT-scanned ETA model with OSAHS by using PIV technique. Four representative phases were highlighted to clarify the respiratory flow patterns and the associated flow structures in the ETA model.

With realistic non-sinusoidal respiratory flow rate and CT-scanned ETA model, the respiratory flow patterns at the reversal phases can neither be regarded as the pattern of inspiration nor that of expiration.

Flow patterns at the inspiratory and expiratory phases are very different. High-speed jet flows generally appear at the opposite sides of the oropharyngeal, laryngopharyngeal and tracheal regions at the inspiration and expiration phases. The expiration process is not a simple reversal process of inspiration.

The respiratory flow patterns and the associated flow structures have clinical implications for breathing tract blockage. The area of stenosis is apt to be blocked at the peak inspiration phase due to potential wall collapse.

To the best of the authors' knowledge, this has been the first research devoted to measuring oscillatory respiratory flows inside CT-scanned ETA model with OSAHS. The PIV measurements of unsteady flow velocity will be used to calibrate the computational fluid dynamics method, which can be further used to capture even more detailed flow fields.

Author Contributions: Funding acquisition, C.Z., Y.J.; project administration, Y.J.; formal analysis, Z.Z.; investigation, Z.Z.; data curation, Z.Z.; writing-original draft preparation, Z.Z.; writing-review and editing, Y.J. and C.Z. All authors have read and agreed to the published version of the manuscript.

Funding: This research was financially supported by the National Key Research and Development Project of China under Grant No. 2016YFB0200901, National Natural Science Foundation of China under Grant No. 51176146, and Shaanxi Key Research and Development Project under Grant No. 2018KWZ-01.

Conflicts of Interest: The authors declare that for this study they have no conflicts of interest.

\section{References}

1. Kesson, A.M. Respiratory virus infections. Paediatr. Respir. Rev. 2007, 8, 240-248. [CrossRef]

2. Lavie, L.; Lavie, P. Molecular mechanisms of cardiovascular disease in OSAHS: The oxidative stress link. Eur. Respir. J. 2009, 33, 1467-1484. [CrossRef] [PubMed]

3. Nithiarasu, P.; Sazonov, I.; Yeo, S.Y. Scan-Based Flow Modelling in Human Upper Airways. In Patient-Specific Modeling in Tomorrow's Medicine; Springer: Berlin/Heidelberg, Germany, 2011; pp. 241-280. 
4. Huang, E.I.; Huang, S.Y.; Lin, Y.C.; Lin, C.M.; Lin, C.K.; Huang, Y.C.; Su, J.A. Increasing Hypopnea in Sleep Breathing Disturbance Improves Postoperative Oxygen Saturation in Patients with Very Severe Obstructive Sleep Apnea. Appl. Sci. 2020, 10, 6539. [CrossRef]

5. Wu, M.F.; Huang, W.C.; Chang, K.M.; Lin, P.C.; Kuo, C.H.; Hsu, C.W.; Shen, T.W. Detection Performance Regarding Sleep Apnea-Hypopnea Episodes with Fuzzy Logic Fusion on Single-Channel Airflow Indexes. Appl. Sci. 2020, 10, 1868. [CrossRef]

6. Taherian, S.; Rahai, H.; Lopez, S.; Shin, J.; Jafari, B. Evaluation of human obstructive sleep apnea using computational fluid dynamics. Commun. Biol. 2019, 2, 423. [CrossRef] [PubMed]

7. Zhu, Z.; Zhang, C.; Zhang, L. Experimental and numerical investigation on inspiration and expiration flows in a three-generation human lung airway model at two flow rates. Resp. Physiol. Neurobiol. 2019, 262, 40-48. [CrossRef]

8. Liu, Y.; So, R.; Zhang, C.H. Modeling the bifurcating flow in a human lung airway. J. Biomech. 2002, 35, 465-473. [CrossRef]

9. Zhang, C.H.; Liu, Y.; So, R.M.C.; Phan-Thien, N. The influence of inlet velocity profile on three-dimensional three-generation bifurcating flows. Comput. Mech. 2002, 29, 422-429. [CrossRef]

10. Liu, Y.; So, R.M.C.; Zhang, C.H. Modeling the bifurcating flow in an asymmetric human lung airway. J. Biomech. 2003, 36, 951-959. [CrossRef]

11. Lu, M.; Liu, Y.; Ye, J.; Luo, H.Y. Large Eddy simulation of flow in realistic human upper airways with obstructive sleep. Procedia Comput. Sci. 2014, 29, 557-564. [CrossRef]

12. Liu, X.; Yan, W.; Liu, Y.; Choy, Y.S.; Wei, Y. Numerical Investigation of Flow Characteristics in the Obstructed Realistic Human Upper Airway. Comput. Math. Methods Med. 2016, 10. [CrossRef] [PubMed]

13. Song, B.; Li, Y.; Sun, J.; Qi, Y.; Li, P.; Li, Y.; Gu, Z. Computational fluid dynamics simulation of changes in the morphology and airflow dynamics of the upper airways in OSAHS patients after treatment with oral appliances. PLoS ONE 2019, 14, e0219642. [CrossRef] [PubMed]

14. Cui, X.; Wu, W.; Ge, H. Investigation of airflow field in the upper airway under unsteady respiration pattern using large eddy simulation method. Resp. Physiol. Neurobiol. 2020, 279, 103468. [CrossRef] [PubMed]

15. Kim, S.K.; Chung, S.K. An investigation on airflow in disordered nasal cavity and its corrected models by tomographic PIV. Meas. Sci. Technol. 2004, 15, 1090-1096. [CrossRef]

16. Im, S.; Heo, G.E.; Jeon, Y.J.; Sung, H.J.; Kim, S.K. Tomographic PIV measurements of flow patterns in a nasal cavity with geometry acquisition. Exp. Fluids 2013, 55. [CrossRef]

17. Wu, H.; Wang, M.; Wang, J.; An, Y.; Wang, H.; Huang, Y. Direct visualizations of air flow in the human upper airway using in-vitro models. Sci. China Life Sci. 2019, 62, 235-243. [CrossRef] [PubMed]

18. Cui, X.; Gutheil, E. Three-dimensional unsteady large eddy simulation of the vortex structures and the mono-disperse particle dispersion in the idealized human upper respiratory system. J. Aerosol Sci. 2017, 114, 195-208. [CrossRef]

19. Cui, X.; Gutheil, E. Large eddy simulation of the flow pattern in an idealized mouth-throat under unsteady inspiration flow conditions. Resp. Physiol. Neurobiol. 2018, 252-253, 38-46. [CrossRef]

20. Evans, H.B.; Castillo, L. Index-matched measurements of the effect of cartilaginous rings on tracheobronchial flow. J. Biomech. 2016, 49, 1601-1606. [CrossRef]

21. Kim, S.K.; Chung, S.K. Investigation on the respiratory airflow in human airway by PIV.J. Vis. Jpn. 2009, 12, 259-266. [CrossRef]

22. Adler, K.; Brücker, C. Dynamic flow in a realistic model of the upper human lung airways. Exp. Fluids 2007, 43, 411-423. [CrossRef]

23. Janke, T.; Schwarze, R.; Bauer, K. Measuring three-dimensional flow structures in the conductive airways using 3D-PTV. Exp. Fluids 2017, 58. [CrossRef]

24. Janke, T.; Koullapis, P.; Kassinos, S.C.; Bauer, K. PIV measurements of the SimInhale benchmark case. Eur. J. Pharm. Sci. 2019, 133, 183-189. [CrossRef]

25. Wright, S.F.; Zadrazil, I.; Markides, C.N. A review of solid-fluid selection options for optical-based measurements in single-phase liquid, two-phase liquid-liquid and multiphase solid-liquid flows. Exp. Fluids 2017,58. [CrossRef]

26. Bates, A.J.; Schuh, A.; Amine-Eddine, G.; McConnell, K.; Loew, W.; Fleck, R.J.; Woods, J.C.; Dumoulin, C.L.; Amin, R.S. Assessing the relationship between movement and airflow in the upper airway using computational fluid dynamics with motion determined from magnetic resonance imaging. Clin. Biomech. 2019, 66, 88-96. [CrossRef] [PubMed] 
27. Le, T.B.; Moghaddam, M.G.; Woodson, B.T.; Garcia, G.J.M. Airflow limitation in a collapsible model of the human pharynx: Physical mechanisms studied with fluid-structure interaction simulations and experiments. Physiol. Rep. 2019, 7, e14099. [CrossRef] [PubMed]

28. Aycock, K.I.; Hariharan, P.; Craven, B.A. Particle image velocimetry measurements in an anatomical vascular model fabricated using inkjet 3D printing. Exp. Fluids 2017, 58. [CrossRef]

29. Lizal, F.; Jedelsky, J.; Morgan, K.; Bauer, K.; Llop, J.; Cossio, U.; Kassinos, S.; Verbanck, S.; Ruiz-Cabello, J.; Santos, A.; et al. Experimental methods for flow and aerosol measurements in human airways and their replicas. Eur. J. Pharm. Sci. 2018, 113, 95-131. [CrossRef]

30. Raffel, M.; Willert, C.E.; Scarano, F.; Kähler, C.J.; Wereley, S.T.; Kompenhans, J. Particle Image Velocimetry: A Practical Guide, 3rd ed.; Springer International Publishing: Cham, Switzerland, 2018.

31. Vanselow, C.; Stöbener, D.; Kiefer, J.; Fischer, A. Particle image velocimetry in refractive index fields of combustion flows. Exp. Fluids 2019, 60. [CrossRef]

32. Große, S.; Schröder, W.; Klaas, M.; Klöckner, A.; Roggenkamp, J. Time resolved analysis of steady and oscillating flow in the upper human airways. Exp. Fluids 2007, 42, 955-970. [CrossRef]

33. Huang, J.; Zhang, L. Numerical simulation of micro-particle deposition in a realistic human upper respiratory tract model during transient breathing cycle. Particuology 2011, 9, 424-431. [CrossRef]

34. West, J.B. Respiratory Physiology: The Essentials, 10th ed.; Lippincott Williams \& Wilkins: Philadelphia, PA, USA, 2012.

35. Womersley, J.R. Method for the calculation of velocity, rate of flow and viscous drag in arteries when the pressure gradient is known. J. Physiol. 1955, 127, 553. [CrossRef] [PubMed]

36. Beresh, S.J. Comparison of PIV data using multiple configurations and processing techniques. Exp. Fluids 2009, 47, 883-896. [CrossRef]

37. Kim, M.; Collier, G.J.; Wild, J.M.; Chung, Y.M. Effect of upper airway on tracheobronchial fluid dynamics. Int. J. Numer. Methods Biomed. Eng. 2018, 34, e3112. [CrossRef] [PubMed]

38. Wang, Y.; Liu, Y.; Sun, X.; Yu, S.; Gao, F. Numerical analysis of respiratory flow patterns within human upper airway. Acta Mech. Sinica PRC 2009, 25, 737-746. [CrossRef]

39. Jalal, S.; Nemes, A.; Van de Moortele, T.; Schmitter, S.; Coletti, F. Three-dimensional inspiratory flow in a double bifurcation airway model. Exp. Fluids 2016, 57. [CrossRef]

40. Lucey, A.D.; King, A.J.; Tetlow, G.A.; Wang, J.; Armstrong, J.J.; Leigh, M.S.; Paduch, A.; Walsh, J.H.; Sampson, D.D.; Eastwood, P.R.; et al. Measurement, reconstruction, and flow-field computation of the human pharynx with application to sleep apnea. IEEE Trans. Biomed. Eng. 2010, 57, 2535-2548. [CrossRef]

Publisher's Note: MDPI stays neutral with regard to jurisdictional claims in published maps and institutional affiliations.

(C) 2020 by the authors. Licensee MDPI, Basel, Switzerland. This article is an open access article distributed under the terms and conditions of the Creative Commons Attribution (CC BY) license (http://creativecommons.org/licenses/by/4.0/). 